\title{
Resistance is futile: The unwitting insertion of analogical inferences in memory
}

\author{
DAVID A. PERROTT, DEDRE GENTNER, and GALEN V. BODENHAUSEN \\ Northwestern University, Evanston, Illinois
}

\begin{abstract}
Analogical inferences can modify people's understanding, but can this occur even when the inferences are unpalatable? We report two experiments suggesting that this is the case. Participants read a source passage on the role and status of gay people in society. Half then read an analogy describing the historical persecution of left-handers. On a subsequent recognition test, the participants who read the analogy were more likely than the control participants to misrecognize analogical inferences as statements explicitly presented, but the two groups did not differ in recognition rates for other kinds of statements. A priori explicit attitudes toward gays did not moderate these findings, although the participants with more positive attitudes toward gays saw the analogy to left-handers as more sound. Our findings demonstrate that analogical inferences can be seamlessly integrated into mental representations of the target domain even when those inferences are unpalatable; in short, resistance to analogy is futile.
\end{abstract}

A potent feature of analogical reasoning is its power as a tool for knowledge acquisition. Analogies are used in scientific reasoning and problem solving (Dunbar, 1995; Gentner, 1983; Holyoak \& Thagard, 1995) and orient learners to new topics (see, e.g., Loewenstein, Thompson, \& Gentner, 1999). Prior research has focused on the deliberate use of analogy, where learners either discover or are given an analogy, use it to derive new inferences, and accept only those inferences that they deem structurally sound and plausible (Clement \& Gentner, 1991; Spellman \& Holyoak, 1996). Recent research by Blanchette and Dunbar $(1999,2002)$ suggests another intriguing possibility. In their studies, participants read descriptions of a target issue (e.g., legalizing marijuana), followed for half the participants by an analogous situation (e.g., lifting the prohibition on alcohol). On a subsequent recognition test, these participants often misidentified analogical inferences as facts actually presented about the target. Furthermore, this analogical insertion effect was robust across variations in passage length, target issue, and the superficial similarity between source and target.

These findings raise the intriguing possibility (which we call the resistance-is-futile hypothesis) that the analogical insertion effect might occur even when the inferences do not fit with the learner's prior attitudes toward the target. This would suggest that the analogical insertion effect might be a means of knowledge accretion and even of conceptual change, against the learner's will.

We thank Isabelle Blanchette, Sam Day, Art Glenberg, and Brian Ross for helpful comments on a previous version of this article. Correspondence concerning this article should be addressed to D. A. Perrott, TrialGraphix, 216 E. 45th St., New York, NY 10017 (e-mail: davidperrott@alumni.northwestern.edu).
Such a mechanism might help explain some of the shifts from one mental model to another in the course of domain learning (Carey, 1985).

One study that provides a hint of such a possibility is Blanchette and Dunbar (1999). Participants were given a single-item measure of their attitudes toward the target issues immediately after they had read the text. These attitudes did not predict occurrence of the analogical insertion effect, suggesting that insertion of inferences may not be highly sensitive to participants' prior attitudes.

\section{The Attitudinal Congeniality Hypothesis}

Against this, extensive evidence attests that people are strongly motivated to resist information that challenges their own viewpoints, even when they are required to process that information (see, e.g., Dunning, 2001; Kunda, 1990; Lord, Ross, \& Lepper, 1979). For example, people scrutinize information that is compatible with their preferences much less extensively than information that contradicts preferred conclusions (Ditto \& Lopez, 1992). If acceptance of analogical inferences would result in counterattitudinal inferences, individuals may be motivated to bar those inferences by, for example, settling upon a more limited or alternative relational structure that lacks the undesired inference or dilutes its import. The attitudinal congeniality hypothesis predicts that the analogical insertion effect is unlikely to occur for counterattitudinal inferences, because these inferences are less likely to be generated and endorsed in the first place and, therefore, less likely to be incorporated seamlessly into mental representations of the target domain. Although Blanchette and Dunbar's $(1999,2002)$ research on the analogical insertion effect involved controversial issues (e.g., legalization of marijuana), the positions advocated arguably were unlikely to elicit negative responses among the pop- 
ulation studied. ${ }^{1}$ In fact, the attitudes reported in Blanchette and Dunbar (1999) were generally weak and clustered around the midpoint of the attitude scale. Moreover, it is possible that the single self-report item employed did not capture a strong and substantial preexisting attitude toward the target issue but, rather, a skeletal attitude that was simply constructed on the fly in response to the materials that the participants had read immediately beforehand. We sought, in the present research, a stronger test of the resistance-is-futile hypothesis against the attitudinal congeniality hypothesis. Such a test would require that the target issue not only be controversial, but also evoke feelings of resistance (measured before exposure to the analogy and assessed with multiple indicators for greater reliability). Since attitudes toward gay people are typically potent and strongly held (e.g., Herek, 1998), this domain was judged to be appropriate for the investigation of our hypotheses.

\section{Soundness of the Analogy}

A secondary goal of the present research was to investigate whether subjective perception of the soundness of the analogy would influence the analogical insertion effect. In Blanchette and Dunbar's (2002) studies, although most of the participants in the analogy group remembered reading the analogical source passage, the average rate of false alarms to analogical inferences was only $44 \%-67 \%$, suggesting that the analogical insertion effect is important but not inevitable.

There is reason to expect that a moderator of the analogical insertion effect might be participants' sense of the soundness of the analogy. A general tenet of analogy research is that people are more likely to draw and retain inferences from matches that they consider sound (Gentner, Rattermann, \& Forbus, 1993; Spellman \& Holyoak, 1996). Theories of analogy further suggest that if individuals make an analogical comparison but then find that it is not compelling, they are less likely to integrate the analogical inferences into their mental representation of the target domain (Clement \& Gentner, 1991). By obtaining participants' subjective evaluations of the soundness of the analogy, we were able to examine this possibility. We also examined whether attitudes toward the target domain influenced the perceived soundness of the analogy, to check whether participants would find the analogy less compelling to the extent that its implications were counterattitudinal.

\section{Overview of the Present Research}

The target domain we chose was legal and social acceptance of homosexuality as a natural part of biological variation. We measured attitudes toward gay people before exposing participants to a passage entitled "Is it Okay to be Gay?" We then experimentally varied exposure to an analogy concerning the historical persecution of left-handed people. A subsequent recognition memory test consisted of items from the text, along with three types of novel items: analogical inferences, plausible false items, and blatantly false items. After the memory test, all the participants were presented with the direct analogy comparing intolerance toward gay people with intolerance toward left-handed people and were asked to evaluate its soundness, using rating scales adapted from other studies of analogical processing (e.g., Gentner et al., 1993).

The attitudinal congeniality hypothesis leads to the prediction that participants with more positive attitudes toward gay people will deem the analogy more sound and evidence higher rates of false alarms for analogical inferences than will participants with more negative attitudes toward gay people. In contrast, the resistance-isfutile hypothesis predicts that misidentification of analogical inferences will occur irrespective of the extent to which the analogy is subjectively unsound or counterattitudinal.

\section{STUDY 1}

\section{Method}

\section{Participants and Design}

The participants were 77 introductory psychology students (58\% female) participating for course credit. By random assignment, there were 38 participants in the analogy group and 39 in the noanalogy group. ${ }^{2}$ The study employed a $2 \times 4$ mixed-factor design, with exposure to the analogical source (analogy or no analogy) as a between-subjects factor and memory test item type (text, analogical inference, plausibly false, or blatantly false) as a repeated measures factor.

\section{Materials and Procedure}

Attitude measurement. In a prior mass-testing session, participants completed a 15-item scale measuring their feelings toward gay people (Moreno \& Bodenhausen, 2001). A sample item from the scale is "I have to admit that being around gay men and lesbians makes me feel uncomfortable."

Learning phase. The target issue was acceptance of gay people as valued members of society. The text presented arguments for and against according gay people legal parity and examined claims concerning whether they threaten the traditional family structure and whether they contribute positively to society. The source analogue was the historical persecution of left-handed people (see Appen$\operatorname{dix} \mathrm{A})$. It was presented after the target analogue as part of the same passage, as in Blanchette and Dunbar's (2002) study. The participants were told that the purpose of the task was to examine psychological issues surrounding the comprehension of controversial passages, that we would like them to read the passage carefully, and that we would ask them questions about it. We then presented the text on a computer screen, either with or without the analogical source paragraph.

Recognition test. After an unrelated 15-min filler task, we used a standard old/new recognition paradigm, asking the participants to indicate, for each of a series of statements, whether the statement was or was not explicitly presented. They received four different types of items in a single, predetermined random order: five statements from the text, four analogical inferences (described below), four plausible false items (statements not in the text, but which plausibly could have been), and two blatantly false items (statements that were obviously not in the text). The average sentence length was 18 words and did not differ according to item type $[F(3,11)=0.49, p=.70]$. Analogical inferences were derived by mapping statements about the source onto the target; to do so, we took statements from the source paragraph and simply replaced the 
words "left-handed people" with "gay people." Similarly, we constructed the plausible false items by modifying statements from the original text, replacing short phrases from these statements with new, but plausible, material (see Appendix B for a list of all analogical inferences and samples of each of the other item types). Four separate measures were derived from the participants' recognition responses by computing the proportion of old answers separately for each item type.

Analogical soundness ratings. Finally, all the participants were presented with the analogical source paragraph and answered four questions assessing analogical soundness (see Appendix B). Demographic information was then obtained from the participants, and they were debriefed.

\section{Results}

\section{Misidentification of Analogical Inferences}

The analogical insertion effect would be evident if exposure to the analogy increased the proportion of old responses to analogical inferences. In accord with this prediction, the participants in the analogy group incorrectly recognized analogical inferences more frequently than did those in the no-analogy group $[M=.35, S D=.33$, vs. $M=.15, S D=.24$; see Figure $1 ; t(76)=3.02, p=.003$ ] The groups did not differ in their proportions of old responses to text items (hits) $[t(76)=0.35, p=.73]$, plausible false items $[t(76)=0.50, p=.62]$, or blatantly false items $[t(76)=0.74, p=.46]$. Thus, the participants in the analogy group were not simply more likely to respond old to all types of items; rather, the effect of being presented with the analogical source was specific to analogical inferences. A $4 \times 2$ mixed ANOVA, with item type as a repeated measure and experimental condition as a between-subjects factor, confirmed a significant interaction between condition and item type $[F(3,228)=4.97$, $\left.M S_{\mathrm{e}}=0.048, p=.002\right]$.

\section{Perceived Analogical Soundness}

The four items measuring the soundness of the analogy were averaged into a composite index $(\alpha=.96)$ that could range from 1 (not at all sound) to 10 (very sound). Actual scores for the participants in the analogy group ranged from 1 to $10(M=5.89, S D=2.68)$. The cutoff for the lower and upper quartiles was 3.63 and 8.31, respectively. Among the participants exposed to the analogy, we expected that those who deemed it more sound would be more likely to misrecognize analogical inferences (but not other types of items) than would those who deemed it to be less sound (i.e., for the analogy group participants, a main effect of soundness of the analogy on the proportion of old responses to analogical inferences). The proportion of old responses to analogical inferences was regressed on rated soundness of the analogy. Contrary to our prediction, soundness did not predict the likelihood of occurrence of the analogical insertion effect $[\beta=.02 ; t(36)=0.72, p=.48]$.

\section{Relationship Between Attitudes and Analogical Processing}

Attitudes toward gay people. The participants' responses to the 15-item scale measuring attitudes toward gay people were averaged into a composite index that could range from 1 (very negative attitude) to 7 (very positive attitude). Actual scores for the participants in the analogy group ranged from 1.8 to $6.8(M=4.69, S D=$ 1.40 ). The cutoff for the lower and upper quartiles was 3.32 and 5.8, respectively. This indicates that attitudes varied considerably toward the target issue of tolerance toward gay people.

Misrecognition of analogical inferences. According to the attitudinal congeniality hypothesis, participants with more positive attitudes toward gay people should be more likely to incorrectly recognize analogical inferences than should participants with more negative attitudes. In contrast, the resistance-is-futile prediction was that attitudes would have no effect.

The proportion of old responses to analogical inferences for those in the analogy group was regressed on at-

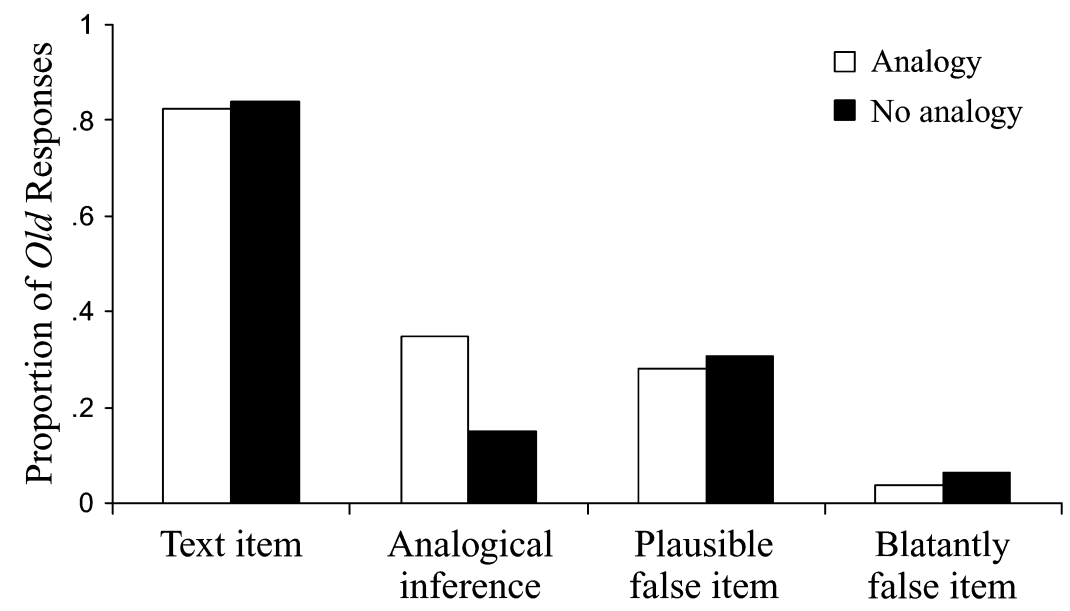

Figure 1. Proportion of old responses, as a function of exposure to the analogical source and item type. 
titudes toward gay people. Consistent with the resistanceis-futile hypothesis, but not the attitudinal congeniality hypothesis, attitudes toward gay people did not predict the likelihood of occurrence of the analogical insertion effect $[\beta=-.01 ; t(27)=-0.21, p=.84]$.

Perceived analogical soundness. As was hypothesized, the analogy group participants with more positive attitudes toward gay people rated the analogy as more sound $[\beta=.94 ; t(27)=2.83, p=.01]$.

\section{STUDY 2}

The results of Study 1 demonstrate that the analogical insertion effect occurs even in the context of an affectively charged social issue and that, once analogical inferences are drawn, it becomes difficult to determine explicit versus implied assertions, even when those assertions are attitudinally unpalatable. An alternative explanation of our findings, however, is that the elevated false alarm rates for analogical inferences stemmed from their being higher in surface similarity to the initial text than were the plausible and the blatantly false items. Study 2 addressed this issue by pairing each (true) analogical inference with a false inference of equally high surface similarity to statements from the source analogue.

\section{Method}

\section{Participants and Design}

Forty-four introductory psychology students (50\% female) participated for course credit. ${ }^{3}$ By random assignment, there were 23 participants in the analogy group and 21 in the no-analogy group. The study employed a $2 \times 5$ mixed-factor design, identical to that in Study 1, except for the addition of the mock (surface-similar) false inferences.

\section{Materials and Procedure}

The materials and procedure were identical to those in Study 1, except that (1) we did not have access to prior attitude scores and (2) four high-surface-similarity mock inferences were added to the recognition test. These were created by making minimal wording changes to the four actual analogical inferences (see Appendix C). Thus, the recognition test consisted of the 11 noninferential items from Study 1, plus four actual and four mock analogical inferences. These were divided across two blocks, with order counterbalanced. Each block contained items of each type, in a single predetermined random order. To minimize carryover effects, items were distributed such that no actual-mock pair was contained in the same block.

\section{Results and Discussion}

If the analogical insertion effect is indeed due to encoding and later misidentification of analogical inferences, the participants in the analogy group should have higher false alarm rates than should those in the no-analogy group to actual, but not to mock, analogical inferences. Alternatively, if the analogical insertion effect in Study 1 was due simply to the high surface similarity between new analogical inferences and old statements from the source analogue from which they were derived (which the participants in the no-analogy group did not see), the analogy group should show elevated false alarms, relative to the no-analogy group, to both mock inferences and actual inferences; that is, there should be a simple main effect of exposure to the analogy.

As was predicted by the analogical insertion effect, but not by the surface similarity account, the participants in the analogy group responded old to actual analogical inferences more frequently than did those in the no-analogy group $[M=.39, S D=.24$, and $M=.23, S D=.26$; $t(42)=2.20, p=.03$ ], but the groups did not differ in their proportion of old responses to the corresponding mock analogical inferences of equally high surface similarity $[M=.09, S D=.12$, and $M=.10, S D=.12 ; t(42)=$ $-0.22, p=.83]$.

As in Study 1, there were no differences across groups in the proportion of old responses to text items $[M=.86$, $S D=.20$, and $M=.77, S D=.26 ; t(42)=1.22, p=.23]$, plausible false items $[M=.30, S D=.23$, and $M=.38$, $S D=.23 ; t(42)=-1.11, p=.27]$, or blatantly false items $[M=.07, S D=.17$, and $M=.00, S D=.00 ; t(42)=1.73$, $p=.09]$. A $5 \times 2$ mixed ANOVA, with item type as a repeated measure and experimental condition as a betweensubjects factor, confirmed a significant interaction between condition and item type $\left[F(4,168)=2.52, M S_{\mathrm{e}}=\right.$ $0.037, p=.04]$.

These results argue against surface similarity between analogical inferences and statements from the source analogue as a viable explanation for the analogical insertion effect found in Study 1.

\section{GENERAL DISCUSSION}

Our findings support the resistance-is-futile hypothesis. They suggest the incorporation of analogical inferences as facts actually presented, even in the context of a highly affectively charged social issue (i.e., tolerance of gay people) where the inferences are attitudinally unpalatable. The results of Study 1 show that the analogical insertion effect is not due simply to the semantic congruence of analogical inferences to statements in the target passage, since semantically congruent noninferential statements (i.e., the plausible false items) were not misrecognized to the same degree. The results of Study 2 show that the analogical insertion effect is not due simply to the surface similarity of actual analogical inferences to statements in the source passage, since mock analogical inferences of equivalent surface similarity were not misrecognized to the same degree.

However, attitudinal congeniality was not entirely impotent. Analogies were deemed more sound the more they jibed with attitudes toward the target domain. Although theories of analogy emphasize the importance of structural consistency and systematicity in the appreciation of analogy (Gentner, 1983; Gentner \& Markman, 1997), we found that greater soundness was not associated with a greater likelihood of misidentifying inferences as presented facts. That is, in the present sample, the analogical insertion effect appeared to be just as likely to occur for unpalatable and/or uncompelling in- 
ferences as it was for palatable and/or compelling ones. Our initial findings with respect to soundness were correlational, and more research will be needed to pin down these processes, but they extend the work of Blanchette and Dunbar (2002) in suggesting that analogical inferences can be integrated unknowingly into mental representations of the target domain even when those inferences are undeniably unpalatable. Our findings, like those of Blanchette and Dunbar (2002), are particularly striking in that the analogical source was presented after the target material (although in the same passage), suggesting that inferences can be inserted into an existing encoding of a target domain.

We have argued that inferences stemming from analogical insertion occur online, during processing of the analogy. Although we cannot refute the possibility that the inferences occurred only during testing, our findings accord with evidence from reading time studies with expository analogies, which document that analogical inferences occur online, during initial processing of a target passage (Blanchette \& Dunbar, 2002, Experiment 4; Day \& Gentner, in press).

Although analogy is often used as a persuasive device in such arenas as politics and marketing, detailed investigation of when and how it is effective is only beginning. Thagard and Shelley (2001) have discussed the use of analogy in transferring and generating emotions and have suggested that emotional coherence is a prime determinant of affective transfer. This raises the question, what are the constraints on analogical persuasion? We found evidence that, even when the target is read before the source analogue, people can be led to make affectively uncongenial inferences and to incorporate them into memory for the target. It remains to be investigated whether this insertion of inferences leads to an attitudinal shift, such that people become more likely to endorse the information. If there are, indeed, aspects of analogical mapping that can influence cognitive processing independently of individuals' attitudes and values, the prospects for using analogies to overcome resistance to persuasion will warrant further exploration.

\section{REFERENCES}

Blanchette, I., \& Dunbar, K. (1999). Memory for analogies and analogical inferences. In M. Hahn \& S. C. Stoness (Eds.), Proceedings of the Twenty-First Annual Conference of the Cognitive Science Society (pp. 73-77). Mahwah, NJ: Erlbaum.

Blanchette, I., \& Dunbar, K. (2002). Representational change and analogy: How analogical inferences alter representations. Journal of Experimental Psychology: Learning, Memory, \& Cognition, 28, 672-685.

CAREY, S. (1985). Conceptual change in childhood. Cambridge, MA: MIT Press.
Clement, C., \& Gentner, D. (1991). Systematicity as a selection constraint in analogical mapping. Cognitive Science, 15, 89-132.

DAY, S. B., \& GENTNER, D. (in press). Nonintentional analogical inference in text comprehension. Memory \& Cognition.

DitTo, P., \& Lopez, D. (1992). Motivated skepticism: Use of differential decision criteria for preferred and nonpreferred conclusions. Journal of Personality \& Social Psychology, 63, 568-584.

DUNBAR, K. (1995). How scientists really reason: Scientific reasoning in real-world laboratories. In R. J. Sternberg \& J. E. Davidson (Eds.), The nature of insight (pp. 365-395). Cambridge, MA: MIT Press.

DunNing, D. (2001). On the motives underlying social cognition. In A. Tesser \& N. Schwarz (Eds.), Blackwell handbook of social psychology: Intraindividual processes (pp. 348-374). Malden, MA: Blackwell.

GentNer, D. (1983). Structure-mapping: A theoretical framework for analogy. Cognitive Science, 7, 155-170.

Gentner, D., \& MarkMAN, A. B. (1997). Structure mapping in analogy and similarity. American Psychologist, 52, 45-56.

Gentner, D., Rattermann, M. J., \& Forbus, K. D. (1993). The roles of similarity in transfer: Separating retrievability from inferential soundness. Cognitive Psychology, 25, 524-575.

HeREK, G. (ED.) (1998). Stigma and sexual orientation: Understanding prejudice against lesbians, gay men, and bisexuals. Thousand Oaks, CA: Sage.

Holyoak, K., \& Thagard, P. (1995). Mental leaps: Analogy in creative thought. Cambridge, MA: MIT Press.

Kunda, Z. (1990). The case for motivated reasoning. Psychological Bulletin, 108, 480-498.

LoEWEnStein, J., ThOMPSOn, L., \& Gentner, D. (1999). Analogical encoding facilitates knowledge transfer in negotiation. Psychonomic Bulletin \& Review, 6, 586-597.

LORD, C., Ross, L., \& LEPPER, M. (1979). Biased assimilation and attitude polarization: The effects of prior theories on subsequently considered evidence. Journal of Personality \& Social Psychology, 37, 2098-2109.

Moreno, K., \& Bodenhausen, G. (2001). Intergroup affect and social judgment: Feelings as inadmissible information. Group Processes \& Intergroup Relations, 4, 21-29.

Sourcebook of criminal justice statistics [Online] (2001). Washington, DC: U.S. Department of Justice, Bureau of Justice Statistics. Available at http://www.albany.edu/sourcebook/1995/pdf/ t285.pdf.

Spellman, B., \& HolyoaK, K. (1996). Pragmatics in analogical mapping. Cognitive Psychology, 31, 307-346.

Thagard, P., \& Shelley, C. (2001). Emotional analogies and analogical inference. In D. Gentner, K. J. Holyoak, \& B. N. Kokinov (Eds.), The analogical mind: Perspectives from cognitive science (pp. 335362). Cambridge, MA: MIT Press.

\section{NOTES}

1. College students generally favor the legalization of marijuana (Sourcebook of Criminal Justice Statistics, 2001). The other issue investigated was destruction of the smallpox vaccine.

2. Data were collected over two academic quarters. Attitudes were measured only for participants taking part during the second quarter of data collection (for 60 of the 77 participants in the total sample; analogy group, $n=30$; no-analogy group, $n=30$ ). The experimental procedure was otherwise identical between the two groups.

3. Two participants were excluded prior to analysis, since the experimenter reported that they had rushed through the experiment and had responded capriciously. 


\section{APPENDIX A \\ Is It Okay to Be Gay?}

Few issues in our society have generated more controversy than the topic of homosexuality. As gay men and lesbians push for marriage rights, adoption rights, and the right to open, unrestricted military service, many people feel conflicted and uncertain about what is best for our society. In order to make informed decisions about these controversial issues, it is more important than ever to understand the nature of homosexuality. What characteristics are typical of gay men and lesbians? What consequences does this type of sexual orientation have for being a productive and valuable member of society? Psychologists, biologists, politicians, theologians, and many others have made strong claims about these issues. These claims need to be closely scrutinized.

A cornerstone of our democratic society is the idea that all citizens should have equal rights and equal protection under the law. Some have argued that, just as ethnic and religious minorities should have legal protections, so should gay men and lesbians. Others argue just as vehemently that not all social groups are in fact equal; they claim that it is a mistake to view gay people as being similar to other social groups. They argue that gay people tend to engage in socially undesirable patterns of behavior that should not be condoned by granting homosexuals equal rights under the law. For example, some have claimed that gay men have a pronounced tendency toward predatory sexual behavior, often targeting underage teens as their victims. This idea has been strongly criticized as inaccurate and stereotypic. These critics have provided evidence that the overwhelming majority of cases of sexual abuse of children are perpetrated by heterosexual men; yet, no one would recommend that male heterosexuals be denied equal rights under the law.

A related concern is the notion that gay people threaten the stability of society by undermining the traditional family structure. From this perspective, the conventional pattern of monogamous heterosexual marriage and child-rearing is seen as the ideal way to promote a stable and prosperous society. Indeed, many of our cultural traditions are built around the institution of marriage and the traditional nuclear family. It is argued that gay people threaten this arrangement by pursuing sexual activity in a manner that is not connected to bonds of committed monogamy and that, in any case, does not provide for procreation. Critics counter that gay men and lesbians constitute a small minority of the population; thus, if gay people fail to pursue monogamous relationships and have children, they in no way prevent the heterosexual majority from following this traditional pathway. These critics argue that the stability of traditional families is threatened much more by societal ills such as economic stress, alcoholism, and drug abuse than it would be by granting gay people equal rights under the law.

Many people believe that gay men and lesbians contribute positively to our society in a variety of ways. For example, gay people are often said to make creative contributions to our society. Indeed, many famous writers, painters, actors, and designers have been gay. Some have argued that the experience of being different from most others may provide a special opportunity to see things from new perspectives, perhaps thereby fueling the fires of creativity. However, critics argue that the "creativity" associated with gay people is often deviant in nature and runs counter to the values of most members of our society. They argue that controversial art and photography exhibits provide evidence that creativity is not necessarily a good thing, if it undermines the cherished values and moral principles of a culture. Clearly, there is much controversy about how we should think about the issue of homosexuality.

\section{[Analogical Source Passage]}

Some people have suggested that this situation can be compared to the way left-handed people were treated in earlier times. Previously, people who were left-handed were suspected of being evil and corrupt. Indeed, the word "sinister" comes from the Latin word for "left." People often failed to realize that handedness is something over which we have very little control. Left-handed people have been persecuted simply because of an irrational fear of human differences. In fact, they have even been forced to eat, write, etc., in a righthanded manner. Now we understand that left-handedness is simply a natural part of the biological variation of our species. From this point of view, although left-handed people are different in certain ways, these differences are easily accommodated in society. 


\section{APPENDIX B}

\section{Description of Source Analogue and Corresponding Inferences for Study 1}

(Word changes to transform the source analog into the corresponding inference are indicated parenthetically.)

People often failed (fail) to realize that handedness (sexual orientation) is something over which we have very little control.

Left-handed (Gay) people have been persecuted simply because of an irrational fear of human differences.

Now we understand that left-handedness (sexual orientation) is a natural part of the biological variation of our species.

Although left-handed (gay) people are different in certain ways, these differences are easily accommodated in society.

\section{Samples of Other Item Types for Study 1}

Sample correct item: Many famous writers, painters, actors, and designers have been gay.

Sample plausible false item: A cornerstone of our democratic society is the idea that marriage and family are institutions that should be strongly protected.

Sample blatantly false item: Contrary to the claims of a few gay rights activists, most gay people are not particularly interested in the topic of equal rights.

\section{Items Assessing Analogical Soundness for Study 1}

(An 11-point response scale was used. Item anchors are indicated parenthetically.)

How well does the history of how left-handed people were viewed apply to the way gay people have been viewed? (very poorly-very well)

How sound is this analogy? That is, how well do the facts about left-handedness fit when applied to the case of homosexuality? (not at all sound-very sound)

How persuasive is the analogy? (not at all persuasive-highly persuasive)

How convinced were you by this argument? (not at all convinced-very convinced).

\section{APPENDIX C \\ Description of Actual and Mock Analogical Inferences for Study 2}

(Word changes to transform true analogical inferences into the corresponding false inferences are indicated parenthetically.)

People often fail to realize that sexual orientation is something over which we have (very little) / (considerable) control.

Gay people have (often) / (never) been persecuted simply because of an irrational fear of human differences.

Now we understand that gayness is (a natural) / (an unnatural) part of the biological variation of our species.

Gay people are different in certain ways, (but) / (and) these differences (are) / (aren't) easily accommodated in society.

(Manuscript received September 4, 2003;

revision accepted for publication October 5, 2004.) 